\title{
ON THE PLAUSIBILITY OF PSYCHOTIC HALLUCINATIONS
}

\author{
Paul Franceschi * \\ University of Corsica, Corte, France
}

\begin{abstract}
In this paper, we describe several factors that can contribute, from the patient's viewpoint, to the plausibility of psychotic hallucinations. We sketch then a Plausibility of Hallucinations Scale, consisting of a 50-item questionnaire, which aims at evaluating the degree of plausibility of hallucinations. We also emphasize the utility of pointing out to the patient the several factors that contribute to the plausibility of his/her hallucinations, in the context of cognitive therapy for schizophrenia.
\end{abstract}

Key words: Schizophrenia; Hallucinations; Cognitive therapy

Cognitive therapy of hallucinations is part of cognitive therapy for schizophrenia. Several accounts of cognitive therapy of hallucinations have been described in the literature (Chadwick et al.,1996; Rector \& Beck, 2002; Kingdon \& Turkington, 2005). On the one hand, Chadwick et al. (1996) stress the importance of the ABC model for cognitive therapy of hallucinations: the hallucinations are the activating events, which engender cognitions, which in turn yield emotional distress and anger. By working on beliefs about the voices, they primary aim at reducing the negative emotions which are the consequences of automatic thoughts following the occurrence of hallucinations. Chadwick et al. also have a special emphasis on the omnipotence and omniscience of the voices. On the other hand, Kingdon \& Turkington (2005) propose the cognitive model of hallucinations as an alternative explanation for the voices: auditory hallucinations are the patient's automatic thoughts that are perceived as originating from outside the patient's mind. Kingdon \& Turkington weigh the available evidence for both competing explanations and finally work on reattribution of auditory hallucinations. Rector \& Beck (2002) take a similar stance, and stress that the final aim of the therapy "is to help patients recognize that the voices simply reflect either their own attitudes about themselves or those they imagine others to have about them".

The purpose of the present paper is to contribute to cognitive therapy for schizophrenia by focusing on the plausibility of psychotic hallucinations. Our concern will be with providing an account of complex hallucinations encountered in schizophrenia that stresses multiple factors which reinforce, from the patient's viewpoint, the intrinsic plausibility of the hallucinations. The purpose of this paper is then to expose how hallucinations can seem plausible and credible to the patient. In section 1, we describe several factors that contribute to the plausibility of hallucinations occurring in schizophrenia. We sketch then in section 2 a scale which is designed to measure accurately the plausibility of hallucinations. In section 3 ,

*Correspondence to: Paul Franceschi, email: p.franceschi@univ-corse.fr

Received May 4, 2011; accepted May 20, 2011; Act Nerv Super (Praha) 53(1-2), 35-43. 
we point out what could be the impact on cognitive therapy for schizophrenia of the present account. Finally, we point out the limitations of the present study and some directions for further research.

\section{FACTORS OF PLAUSIBILITY OF HALLUCINATIONS}

We shall enumerate in what follows several factors that can contribute, from the patient's viewpoint, to the plausibility of the hallucinations that he/she experiences. Hallucinations are one major symptom of schizophrenia. According to DSM-IV, a hallucination is defined as "A sensory perception that has the compelling sense of reality of a true perception but that occurs without external stimulation of the relevant sensory organ". (DSM-IV, p. 767). By plausibility, we mean the fact that the patient's abnormal perceptions are seemingly attributable to an external source (usually, other people). The plausibility that results from certain phenomenological features of auditory hallucinations has notably been hinted at by Stephane et al. (2003):

(...) hearing "multiple voices" is associated with attribution of the "voices" to others, which is plausible intuitively as well. This indicates that the patients' experiences of hallucinations could be understood, intuitively, based on common sense experiences of the world.

In this paper, we shall expand this idea, by pointing out that multiple factors are susceptible of congruently strengthening the patient's conviction that his/her abnormal perceptions come from the external world.

Hallucinations come in a variety of modalities. In order to shed light on the factors that can lead to the plausibility of hallucinations occurring in schizophrenia, it is worth drawing first some useful distinctions.

\subsection{Unimodal and multi-modal hallucinations}

Let us consider, to begin with, the distinction between unimodal and multi-modal hallucinations. Unimodal hallucinations can be classified into five types, corresponding to our five sensory pathways: auditory, visual, olfactory, tactile and gustatory. Multi-modal hallucinations are made up of unimodal hallucinations of different types which occur simultaneously (or quasi-simultaneously). There are accordingly 26 combinations of multimodal hallucinations (plus 5 unimodal ones). The latter can be enumerated exhaustively as follows (we also describe an instance of some common multi-modal cases, since it can be useful for explanatory purposes):

(i) 1-modal: auditory, visual, olfactory, tactile, gustatory

(ii) 2-modal: auditory-visual ("I saw $x$ sitting on my bed and I heard him saying 'Bastard!'”), auditory-olfactory ("I heard $x$ saying 'I will smoke a cigar!' and at this very moment I smell a taste of tobacco"), auditory-tactile ("I heard $x$ saying 'You will be stung by a scorpion!' and at this very moment I felt a sharp sting of pain on my left arm"), auditory-gustatory, visualolfactory ("I saw $x$ on my bed smoking a cigar and I also smell the taste of tobacco"), visualtactile ("I saw a scorpion on my left arm and at this very moment I felt a sharp sting of pain there"), visual-gustatory ("I saw blood dripping from my finger and it had the taste of blood when I put it on my tongue"), olfactory-tactile, olfactory-gustatory, tactile-gustatory

(iii) 3-modal: auditory-visual-olfactory, auditory-visual-tactile ("I heard $x$ saying 'You will be stung by a scorpion!' and at this very moment I saw a scorpion on my left arm while feeling a sharp sting of pain there"), auditory-visual-gustatory ("I heard 'I will harm you' and at this very moment I saw blood dripping from my finger and it had the taste of blood when I put it on my tongue"), auditory-olfactory-tactile, auditory-olfactory-gustatory, auditorytactile-gustatory, visual-olfactory-tactile, visual-olfactory-gustatory, visual-tactile-gustatory, olfactory-tactile-gustatory 
(iv) 4-modal: auditory-visual-olfactory-tactile, auditory-visual-olfactory-gustatory, auditoryvisual-tactile-gustatory ("I heard 'I will harm you' and then I saw blood dripping from my finger, while I felt a sharp pain there. It had the taste of blood when I put it on my tongue"), auditory-olfactory-tactile-gustatory, visual-olfactory-tactile-gustatory

(v) 5-modal: auditory-visual-olfactory-tactile-gustatory

At this step, it should be noted that multi-modal hallucinations retain their force from the plausibility that results from the simultaneous (or quasi-simultaneous) occurrence of two or more unimodal hallucinations of different types. For this reason, multi-modal hallucinations retain considerable power with regard to unimodal ones. The sense of reality that results from multi-modal hallucinations is due to the fact that several sensory pathways are congruently involved in the hallucinatory process. If we consider, for example, bimodal hallucinations of the auditory-visual type, it proves to be that the simultaneous occurrence of an additional visual hallucination strongly reinforces the sense of reality that results from the auditory hallucination. As the above examples illustrate, multi-modal hallucinations are seemingly highly more plausible and realistic than unimodal ones, and result in a much greater sense of realism. More generally, it illustrates how $(n+1)$-modal hallucinations are seemingly much more realistic than $n$-modal ones, a supplementary sensory pathway being involved in the hallucinatory process.

\subsection{Factors of plausibility of auditory hallucinations}

It is worth mentioning, second, several factors that can contribute to the intrinsic plausibility of auditory hallucinations:

(i) structured versus unstructured auditory hallucinations: structured sounds notably consist of comments on the patient's thoughts or activities, conversations of several persons, or commands ordering the patient to do things, etc., while on the other hand, unstructured sounds consist of ringing, buzzing, whistling, etc.

(ii) auditory hallucinations having an external versus an internal origin: auditory hallucinations seemingly coming out from outer space could reinforce the idea that the voices have an external origin, e.g. are attributable to other people.

(iii) the locus (Chadwick et al., 1996, p. 103) - i.e. the space location - of auditory hallucinations is also susceptible of reinforcing their intrinsic plausibility. We can consider, for example, a patient who hears the voice of the presenter of the show saying 'Bastard!'. Now this sounds more realistic if the locus of the voice is the television device rather than the ashtray. Let us suppose now that the patient hears a voice saying 'I can read your thoughts'. Now it sounds more likely to the patient if the voice comes out from the telephone than from the halogen lamp.

It is worth noting here that this criterion is susceptible to vary from culture to culture. ${ }^{1}$ In effect, depending on the individuals, a speaking tree or a speaking animal could be, in certain cases, consistent with the patient's cultural background.

(iv) bilateral versus unilateral auditory hallucinations: auditory hallucinations coming indifferently from the patient's right or from his/her left are more plausible than unilateral ones. $^{2}$

(v) time location related versus unrelated to the patient's thoughts, emotions or actions (Stephane et al. 2003 make mention of the "relation to the moment"). In this regard, auditory hallucinations that are simultaneous with the patients internal phenomena gain more plausibility.

(vi) phrases versus single words: in this context, phrases, conversations, elaborate sentences are more plausible than single words.

\footnotetext{
${ }^{1}$ We thank Paul Gilbert for the suggestion of taking into account cultural beliefs with regard to this specific criterion.

2 We owe the suggestion to include the bilateral/unilateral distinction related to hallucinations in all modalities to Peter Brugger.
} 
(vii) multiple voices versus single voice (Stephane et al., 2003).

(viii) auditory hallucinations fitting versus not fitting with the patient's desires or fears: this factor consists of whether the hallucinations experienced by a patient fit adequately or not with his/her individual fears or desires. For in the affirmative, it would greatly increase the plausibility of the corresponding hallucinations. Let us take an example. The patient is very anxious about the evolution of his/her illness. He/she hears a voice that says: 'You will relapse next month'. Now the content of this auditory hallucination fits adequately with the patient's own fears. The reason why auditory hallucinations fitting with desires or fears are more plausible, is that they are coherent with the patient's belief system. By contrast, had the content of auditory hallucinations been unrelated or contradictory with the patient's desires and fears, the corresponding information would have then resulted in a lack of coherence with the patient's belief system (this is in line with the approach to hallucinations exposed in Rector \& Beck, 2002, which is concerned with: "(...) how the specific voice content and beliefs about the voices reflect the person's prehallucinatory fears, concerns, interests, preoccupations and fantasies").

(ix) interactive versus non-interactive voices: whether the patient can interact or not with voices, i.e. discuss or engage in dialog with them.

\subsection{Factors of plausibility of visual hallucinations}

Several factors can contribute, third, to the intrinsic plausibility of visual hallucinations:

(i) formed versus unformed visual hallucinations: formed hallucinations are made up of figures, faces, morphing objects or scenes. By contrast, unformed hallucinations consist of dots, lines, geometrical figures, flashes, etc.

(ii) ordinary versus bizarre or extraordinary visual hallucinations: for obvious reasons, objects that look ordinary gain more likeliness than bizarre, unreal objects.

(iii) objects in color versus in black and white.

(iv) visual hallucinations fitting versus not fitting with surroundings. as noted by Teunisse et al. (1996), the relationship to surroundings could play an important role in the plausibility of complex hallucinations. Such or such unimodal hallucination could fit well (e.g. a person lying on a bed, a scorpion walking on the ground) or not (a figure on the ceiling) with surroundings. Now it should be apparent that fitting with surroundings visual hallucinations are consistent with the patient's knowledge of the physical world. This renders, from the patient's viewpoint, the hallucination very plausible. By analogy with the locus of auditory hallucinations, fitting with surroundings can be assimilated to the locus - i.e. space location of visual hallucinations.

(v) bilateral versus unilateral visual hallucinations.

(vi) time location of visual hallucinations related versus unrelated to the patient's thoughts, emotions or actions (e.g. the patient thinks to a scorpion and at this very moment he/she sees a scorpion on the ground).

(vii) animated versus static images.

\subsection{Factors of plausibility of olfactory hallucinations}

Several factors can contribute, fourth, to the plausibility of olfactory hallucinations:

(i) bilateral versus unilateral olfactory hallucinations.

(ii) olfactory hallucinations fitting versus not fitting with the patient's desires or fears: the patient fears of being killed and smells a poisonous gas in his/her room.

(iii) transient versus permanent olfactory hallucinations: some patients experience olfactory hallucinations that occur any time of day and also last for hours (Tousi \& Frankel 2004). 


\subsection{Factors of plausibility of tactile hallucinations}

Certain factors can contribute, fifth, to the plausibility of tactile hallucinations:

(i) bilateral versus unilateral tactile hallucinations.

(ii) tactile hallucinations fitting versus not fitting with the patient's desires or fears. the patient fears of being murdered and feels an electric-shock sensation.

\subsection{Factors of plausibility of gustatory hallucinations}

Some factors can contribute, sixth, to the plausibility of gustatory hallucinations:

(i) common versus strange gustatory hallucinations: in some cases, the patient may find that his/her food tastes strange. This could decrease the plausibility of the corresponding hallucination, in contrast with gustatory hallucinations where the patient experiences normal and common tastes.

(ii) gustatory hallucinations fitting versus not fitting with the patient's desires or fears: the patient fears of being poisoned and feels the taste of poison in his/her mouth.

\section{PLAUSIBILITY OF HALLUCINATIONS SCALE}

From the above, it results that it could be useful to measure accurately the plausibility of the hallucinations occurring in schizophrenia. For this purpose, we shall now sketch a 50-item scale, which is targeted at evaluating the plausibility of hallucinations experienced by a patient. This binary scale consists of a questionnaire which allows for yes/no answers (each yes answer weighting 2 points).

Table 1. Hallucinations scale.

\begin{tabular}{|l|l|}
\hline Item & Questions (0-50) \\
\hline & Unimodal hallucinations \\
\hline & Auditory hallucinations \\
\hline $\mathbf{1}$ & Does the patient hear auditory hallucinatory which consist of structured sounds? \\
\hline $\mathbf{2}$ & Does the patient experience auditory hallucinations which come out from outer space? \\
\hline $\mathbf{3}$ & Does the patient experience auditory hallucinations whose locus sounds realistic? \\
\hline $\mathbf{4}$ & Does the patient experience bilateral auditory hallucinations? \\
\hline $\mathbf{5}$ & $\begin{array}{l}\text { Does the patient experience auditory hallucinations whose time location is related to the patient's } \\
\text { thoughts, emotions or actions? }\end{array}$ \\
\hline $\mathbf{6}$ & Does the patient experience auditory hallucinations which consist of phrases, conversations? \\
\hline $\mathbf{7}$ & Does the patient experience auditory hallucinations with multiple voices? \\
\hline $\mathbf{8}$ & Does the patient experience auditory hallucinations whose content fits with his/her fears or desires? \\
\hline $\mathbf{1 0}$ & Can the patient interact with auditory hallucinations, i.e. discuss or engage in dialog with them? \\
\hline & Visual hallucinations \\
\hline $\mathbf{1 1}$ & Does the patient experience formed visual hallucinations? \\
\hline $\mathbf{1 2}$ & Does the patient experience visual hallucinations with ordinary objects? \\
\hline $\mathbf{1 3}$ & Does the patient experience visual hallucinations in color? \\
\hline $\mathbf{1 4}$ & Does the patient experience visual hallucinations whose locus fits with surroundings? \\
\hline $\mathbf{1 5}$ & Does the patient experience bilateral visual hallucinations? \\
\hline $\mathbf{1 6}$ & $\begin{array}{l}\text { Does the patient experience visual hallucinations whose time location is related to his/her thoughts, } \\
\text { emotions or actions? }\end{array}$ \\
\hline $\mathbf{1 7}$ & Does the patient experience visual hallucinations consisting of scenes or sequences of animated images? \\
\hline
\end{tabular}




\begin{tabular}{|c|c|}
\hline & Olfactory hallucinations \\
\hline 18 & Does the patient experience bilateral olfactory hallucinations? \\
\hline 19 & Does the patient experience olfactory hallucinations whose content fits with his/her fears or desires? \\
\hline \multirow[t]{2}{*}{20} & Does the patient experience transient olfactory hallucinations? \\
\hline & Tactile hallucinations \\
\hline 21 & Does the patient experience bilateral tactile hallucinations? \\
\hline \multirow[t]{2}{*}{22} & Does the patient experience tactile hallucinations whose content fits with his/her fears or desires? \\
\hline & Gustatory hallucinations \\
\hline 23 & Does the patient experience gustatory hallucinations of a common type? \\
\hline \multirow[t]{2}{*}{24} & Does the patient experience gustatory hallucinations whose content fits with his/her fears or desires? \\
\hline & Bimodal hallucinations \\
\hline 25 & Does the patient experience bimodal hallucinations of the auditory-visual type? \\
\hline 26 & Does the patient experience bimodal hallucinations of the auditory-olfactory type? \\
\hline 27 & Does the patient experience bimodal hallucinations of the auditory-tactile type? \\
\hline 28 & Does the patient experience bimodal hallucinations of the auditory-gustatory type? \\
\hline 29 & Does the patient experience bimodal hallucinations of the visual-olfactory type? \\
\hline 30 & Does the patient experience bimodal hallucinations of the visual-tactile type? \\
\hline 31 & Does the patient experience bimodal hallucinations of the visual-gustatory type? \\
\hline 32 & Does the patient experience bimodal hallucinations of the olfactory-tactile type? \\
\hline 33 & Does the patient experience bimodal hallucinations of the olfactory-gustatory type? \\
\hline \multirow[t]{2}{*}{34} & Does the patient experience bimodal hallucinations of the tactile-gustatory type? \\
\hline & Trimodal hallucinations \\
\hline 35 & Does the patient experience trimodal hallucinations of the auditory-visual-olfactory type? \\
\hline 36 & Does the patient experience trimodal hallucinations of the auditory-visual-tactile type? \\
\hline 37 & Does the patient experience trimodal hallucinations of the auditory-visual-gustatory type? \\
\hline 38 & Does the patient experience trimodal hallucinations of the auditory-olfactory-tactile type? \\
\hline 39 & Does the patient experience trimodal hallucinations of the auditory-olfactory-gustatory type? \\
\hline 40 & Does the patient experience trimodal hallucinations of the auditory-tactile-gustatory type? \\
\hline 41 & Does the patient experience trimodal hallucinations of the visual-olfactory-tactile type? \\
\hline 42 & Does the patient experience trimodal hallucinations of the visual-olfactory-gustatory type? \\
\hline 43 & Does the patient experience trimodal hallucinations of the visual-tactile-gustatory type? \\
\hline \multirow[t]{2}{*}{44} & Does the patient experience trimodal hallucinations of the olfactory-tactile-gustatory type? \\
\hline & Quadri-modal hallucinations \\
\hline 45 & Does the patient experience quadri-modal hallucinations of the auditory-visual-olfactory-tactile type? \\
\hline 46 & Does the patient experience quadri-modal hallucinations of the auditory-visual-olfactory-gustatory type? \\
\hline 47 & Does the patient experience quadri-modal hallucinations of the auditory-visual-tactile-gustatory type? \\
\hline 48 & Does the patient experience quadri-modal hallucinations of the auditory-olfactory-tactile-gustatory type? \\
\hline \multirow[t]{2}{*}{49} & Does the patient experience quadri-modal hallucinations of the visual-olfactory-tactile-gustatory type? \\
\hline & Quinti-modal hallucinations \\
\hline 50 & $\begin{array}{l}\text { Does the patient experience quinti-modal hallucinations of the auditory-visual-tactile-olfactory-gustatory } \\
\text { type? }\end{array}$ \\
\hline
\end{tabular}


It is worth noting that this 50 -item scale can be regarded as non-specific to psychotic hallucinations. It is also suited to other disorders or illnesses involving hallucinations. Among these are other mental illnesses, but also Charles Bonnet syndrome (Teunisse et al., 1996; Menon et al., 2003), epilepsy (Sachdev, 1998; Schwartz \& Marsh, 2000), etc. In particular, the Charles Bonnet syndrome occurs in the elderly and is usually associated with ocular pathology and severe visual impairment. The Charles Bonnet syndrome is characterized by the presence of complex and persistent visual hallucinations. The syndrome is usually associated with an absence of hallucinations in other sensory modalities. It is worth noting that the Charles Bonnet syndrome affects psychologically normal individuals with full or partial insight and the patients are accordingly non-delusional. On the other hand, auditory hallucinations are frequently associated with temporal lobe epilepsy, where hallucinations in other modalities can also occur.

It is worth mentioning that the Plausibility of Hallucinations Scale could also be used in association with other instruments for measuring insight, such as the Beck Cognitive Insight Scale (Beck et al., 2003) in order to gain more accurate knowledge of the patient's state. For schizophrenia is usually associated with lack of insight into the internal origin of the hallucinations. By contrast, in other illnesses such as Charles Bonnet syndrome or in pseudohallucinations related to brain trauma or PTSD (Stephane et al., 2004), the patient usually retains insight into the internal origin of his/her hallucinations.

\section{IMPACT ON COGNITIVE THERAPY}

We suggest that the above emphasis on the plausibility of hallucinations could be usefully incorporated into the process of cognitive-behavior therapy of schizophrenia (Kingdon \& Turkington, 1994, 2005; Chadwick et al., 1996; Rector \& Beck, 2002). The general idea would be to point out to the patient who experiences highly plausible hallucinations those factors that confer to his/her hallucinations their intrinsic plausibility. Hopefully, this could insert itself well into the process of cognitive-behavior therapy, whose primary goal is to help the patient gaining more insight into the nature of his/her hallucinations and in particular to understand that they do not originate from an external source. In this context, stressing to the patient the plausibility of his/her hallucinations, could help him/her understand better how hallucinations can be self-deceiving.

It is worth focusing, to begin with, on multi-modal hallucinations. In this context, a first step would be to point out to the patient that multi-modal hallucinations are capable of seeming very plausible and realistic. It could then be argued and explained to the patient that multi-modal hallucinations are more plausible than unimodal ones. This could be illustrated through some examples. This latter strategy could make use of "what if statements" (Ellis \& Dryden, 1997). Along these lines, it could be pointing out to the patient that if someone, instead of experiencing one single auditory hallucination, would experience simultaneously one supplementary visual hallucination, then the resulting multi-modal (of the auditoryvisual type) hallucination would sound much more realistic. Along these lines, it could be pointed out to the patient that the particular case of multi-modal hallucination that he/she experiences is potentially very realistic and inherently capable of deceiving him/her.

Once the patient familiar with the concept of multi-modal hallucinations, another goal could be to learn the patient how to use by himself/herself the preceding taxonomy of multimodal hallucinations and to apply it when he/she experiences these types of complex hallucinations. He/she would then be capable of identifying the corresponding case at hand. Hopefully, this could help the patient rationalize his/her abnormal perceptions and perhaps accept better the internal origin of his/her hallucinations as an alternative explanation.

The fact of classifying multi-modal hallucinations would be helpful to the patient, it seems, to help him/her rationalize and explain the phenomena he/she experiences. For we should bear in mind that the patient experiences abnormal phenomena, which are unfamiliar to psychologically normal people. In this context, helping the patient rationalize, classify and 
describe accurately the phenomena of his/her own internal world, proves then to be a valuable practical goal to attain. Accordingly, identifying, recognizing and labeling a given type of multi-modal hallucination could help lessen its associated omnipotence (Chadwick et al., 1996). This could be helpful to the patient, we suggest, who ordinarily faces an unexplained and upsetting phenomenon. More generally, the fact of identifying the various factors that render his/her complex hallucinations so plausible could help the patient gaining more insight into the internal origin of his/her hallucinations. At this step, it should be noted that the present account is notably in line - for what concerns the delusion that consists in attributing an external origin to the hallucinations - with the views advocated by Brendan Maher $(1988,1999)$, who sees delusions as a patient's attempt to explain some perplexing and puzzling phenomena. According to Maher, delusions arise from normal (mainly rational but occasionally irrational) reasoning applied to abnormal phenomena. Among these abnormal phenomena which are very perplexing to the patient are the hallucinations.

\section{LIMITATIONS AND DIRECTIONS FOR FURTHER RESEARCH}

The main limitation of the present study is that the psychometric properties of the Plausibility of Hallucinations Scale have not been tested. However, given the number of items of the scale, one can expect a good sensibility. On the other hand, the reliability and validity of the scale remain to be tested.

Finally, the above developments suggest several questions, which could be usefully the subject of further study, based on the Plausibility of Hallucinations Scale. A first question that arises is the following: (i) Is the plausibility of hallucinations rate higher in schizophrenia than in other illnesses involving hallucinations, e.g. other mental illnesses, Charles Bonnet syndrome, temporal lobe epilepsy, etc.? A comparison of the plausibility of hallucinations rate occurring in schizophrenia and other illnesses involving hallucinations could be made accordingly. We suggest that such comparison could provide some useful information about the relationships of these illnesses (Sachdev, 1998). Although schizophrenia (paranoid subtype) should prima facie involve a higher rating, it seems that an accurate measure of the degree of plausibility of hallucinations could result in some interesting information. Along these lines, a comparison of the plausibility of hallucinations ratings occurring in different subtypes of schizophrenia could also be informative.

The above Plausibility of Hallucinations Scale is also designed to allow for comparisons between different chronological states in the same patient. This suggests a second type of question: (ii) Does the plausibility of hallucinations rating evolve during the course of schizophrenia? Along these lines, Nayani \& David (1996) observed an increase in the complexity of auditory hallucinations over time, seemingly related to lesser distress and better coping. A similar question could be raised for other illnesses involving hallucinations. In this context, Menon et al. (2003) reported accordingly that "Elementary hallucinations may progressively evolve into complex visual hallucinations" in the Charles Bonnet syndrome.

Lastly, a third interesting question goes as follows: (iii) Is the plausibility rate of hallucinations occurring in schizophrenia correlated with the I.Q. of the patient, i.e. do patients with a high I.Q. more frequently experience complex hallucinations with a high level of plausibility? In other words, is the following hypothesis confirmed: The higher the I.Q., the higher the plausibility of hallucinations rating? Hopefully, the answer to these questions will provide some information that might well be useful to the understanding of the illness and to cognitive-behavior therapy of schizophrenia.

\section{ACKNOWLEDGMENTS}

We thank Peter Brugger, Paul Gilbert and Hélène Verdoux for very useful comments on earlier drafts. 


\section{REFERENCES}

American Psychiatric Association (1994). Diagnostic and Statistical Manual of Mental Disorders (4th edition). American Psychiatric Association: Washington.

Beck, A. T., Rector, N. A. (2003). A Cognitive Model of Hallucinations. Cognitive Therapy and Research, 27(1), 19-52.

Beck, A. T., Baruch, E., Balter, J. M., Steer, R. A., Warman, D. M. (2004). A new instrument for measuring insight: the Beck Cognitive Insight Scale. Schizophrenia Research, 68(2-3), 319-329.

Chadwick, P., Birchwood, M. \& Trower, P. (1996). Cognitive Therapy for Delusions, Voices, and Paranoia. Chichester: Wiley.

Ellis, A., Dryden, W. (1997). The practice of rational emotive behaviour therapy, London: Free Association Books.

Kingdon, D. \& Turkington, D. (1994). Cognitive-behavioural Therapy of Schizophrenia. Guilford: New York.

Kingdon, D., \& Turkington, D. (2005). Cognitive Therapy of Schizophrenia. New York, London: Guilford.

Maher, B. A. (1988). Anomalous experiences and delusional thinking: the logic of explanations. In: T.F. Oltmanns \& B.A. Maher (Eds.), Delusional Beliefs, pp. 15-33. Wiley: New York.

Maher, B. A. (1999). Anomalous experience in everyday life: Its significance for psychopathology. The Monist, 82, 547-570.

Menon, G. J., Rahman, I., Menon, S. J., Dutton, G. N. (2003). Complex visual hallucinations in the visually impaired: the Charles Bonnet Syndrome. Survey of Ophthalmology, 48, 58-72.

Nayani, T. H., David, A. S. (1996). The auditory hallucination: a phenomenological survey. Psychological Medicine, 26(1), 177-189.

Rector, N. A., Beck A. T. (2002). Cognitive Therapy for Schizophrenia: From Conceptualization to Intervention. Canadian Journal of Psychiatry, 47(1), 41-50.

Sachdev, P. (1998). Schizophrenia-Like Psychosis and Epilepsy: The Status of the Association. American Journal of Psychiatry, 155(3), 325-336.

Schwartz, J. M., Marsh, L. (2000). The Psychiatric Perspectives of Epilepsy. Psychosomatics, 41(1), 31-38.

Siddle, R. (2002). Communications from my parents. In: D. Kingdon \& D. Turkington (Eds.), The Case Study Guide to Cognitive Behaviour Therapy of Psychosis, pp. 109-121. Chichester: Wiley.

Stephane, M., Thuras, P., Nasrallah, H., Georgopoulos, A. P. (2003). The internal structure of the phenomenology of auditory verbal hallucinations. Schizophrenia Research, 61, 185-193.

Stephane, M., Hill, T., Matthew, E., \& Folstein, M. (2004) New phenomenon of abnormal auditory perception associated with emotional and head trauma: Pathological confirmation by SPECT scan. Brain and Language, 89, 503-507.

Teunisse, R. J., Cruysberg, J. R., Hoefnagels, W. H. (1996). Visual hallucinations in psychologically normal people: Charles Bonnet's syndrome. Lancet, 347, 794-797.

Toone, B. K. (2000). The psychoses of epilepsy. Journal of Neurology, Neurosurgery and Psychiatry, 69(1), 13. 